\title{
Head trauma and epilepsy as medical risk factors in the development of brain tumors in Albania
}

\begin{abstract}
Summary
Several diseases and medical treatments are discussed as risk factors for the development of brain tumors, including infections, allergy, alterations of immune system, cranial trauma, hormonal factors, epilepsy, cancer family history, etc. We discuss here the role of head trauma and epilepsy as medical risk factor in development of brain tumor

A total 1883 patients with brain tumors are registered from 1993-2013, 977 (49\%) of them were interviewed to review the analysis of risk factors, during the period December 2010-December 2013. We found in our study a correlation between head trauma and brain tumor (meningeoma) around 1\%, not a correlation between glioma and traumatic brain injury (TBI) in male, and no correlation between meningeoma, glioma and TBI in female. The role of epilepsy in development of brain tumors in our study is unclear, this for the stigma that exist in our country on epilepsy, only $0.87 \%$ of patients diagnosed with brain tumors referred to suffer from epilepsy from 10-15 years.
\end{abstract}

The role of medical factors in development of brain tumors is important. The role of head trauma is more evident in male than female in our study and is unclear the role of epilepsy.

Keywords: Head Trauma, Epilepsy, Brain Tumor, Risk Factor
Volume 5 Issue 2 - 2016

\author{
Djamandi P,' Brace G, ${ }^{2}$ Kaloshi G, ${ }^{2}$ Mentor \\ Petrela ${ }^{2}$ \\ 'Hygeia Hospital,Tirana,Albania \\ ¿University Hospital Center "Mother Teresa", Neurosurgery \\ Service, Albania
}

Correspondence: Pavllo Djamandi MD, Hygeia Hospital, Mbikalimi I Kamzes, Mezes Fushe I II,Tirana,Albania,Tel 355692628029, Email pavllo06@gmail.com

Received: May 18, 2016 | Published: November 08, 2016

\section{Introduction}

Tumors of the central nervous system (CNS) are a heterogeneous group of neoplasms that include all forms of primary or secondary neoplasms developed within cranial vertebral cavity. CNS tumors represent about $2 \%$ of all new cancers and the same percentage was reported in European and non-European population. ${ }^{1-3}$ They represent an unusual group neoplasms regarding incidence, however, they represent a common form of death from cancer, representing approximately $3.5 \%$ of the deaths from cancer. ${ }^{4}$ Finally, an increased incidence of tumors of the CNS was reported by many studies. ${ }^{1,5,6} \mathrm{~A}$ part of this increase in incidence can be attributed to improvements of diagnostic techniques; however, probably real growth of brain tumors was attributed to increased number of risk factors and the duration of their exposure.

A risk factor is anything that affects the chance of each individual to be caught by a disease such as brain tumor. We can change several factors, such as smoking, but we cannot change other factors such as age, sex, or family history. But having a risk factor, even some, does not mean that an individual will ever be caught by the disease, and many people may have tumors even without any known risk factor. On the other hand, if a person has a risk factor, it is often very difficult to know how much he can contribute to the development of the brain tumor.

Increased brain tumor risk after head trauma suggested by case reports and clinical series has been previously studied epidemiologically with mixed results. Evidence for elevated brain tumor risk after head trauma was strongest for meningeoma in men. ${ }^{7}$ Most brain tumors have no causal link with any known risk factor and no apparent cause. But there are several factors that may promote the risk of brain tumors.

Seizures has been associated with brain tumors, because a brain tumor can cause seizures, till now is unclearly the role of epilepsy, or if antiepileptic drugs increase the risk of development of brain tumors.

\section{Methods}

A total of 1883 of CNS tumors were registered during this period, from 1993 to 2013. Data from 1993 to 2010 were absorbed by registers in neurosurgery and pathological anatomy services. Of these, 977 cases (49\%) were interviewed, conducted to investigate for consideration the risk factors, during December 2010- December 2013.

Statistical analysis was performed by G. Kaloshi, MD, using JMP 11 software (SAS company). Maximum likelihood estimates odds ratios (OR) and confidence intervals CI - 95\% were computed using both conditional and, to minimize the problem of missing data, unconditional logistic regression. Unconditional logistic regression correlates with the variables included age and gender.

\section{Results}

As seen in Table 1 on the incidence by year, there has been an increased incidence of CNS tumors. Interpretation of this growth is complicated greatly from 2 "top", respectively 1995-1996 years and 2001-2002 periods which coincide with the addition of diagnostic procedures (CT scanner) or improving their (the introduction of MRI). These procedures have been present for a neurosurgeon with changing / improving not only the professional experience of the staff, but also the care diagnostic, therapeutic and even access to patients of all ages. The impact of diagnostic improvement is seen especially in increasing the incidence over the years at the extremes of age, below 10 years of age and over 70 years old. This means that doctors become increasingly willing to evaluate and treat elderly patients by changing the popular paradigms.

Examination of trends in the incidence of gliomas high versus lowgrade glioma observed an increased incidence of convergence in the age group 15-44 years, while divergence seen a "dramatic" increase in the incidence of high grade (increase) those lower (discount trend) in the age group over 45 years (Table 2). 
Although environmental factors may be implicated in some epidemiological studies, to date has not identified any factor impact with absolute risk. For this reason, we are not trying to explain by changes in time quantitative trends of the incidence of brain tumors solely on the basis of environmental factors.

\section{Gender}

Gender constitutes a significant factor risk, especially in the two main types of tumors of the CNS, gliomas or neuro-epithelial tumors and meningeoma (meningeal tumors) in which noted a predominance of males in gliomas and a predominance about "exclusive" women in meningioma. These appear quite clearly in Table 3.

\section{Trauma and head injuries}

For long time head trauma is predisposing or suspected as risk factor for inducing certain types of brain tumors. Epidemiological studies have tried to help determine which types of tumors or not connected to this trauma. Several studies have shown trauma connection with glioma, ${ }^{8}$ with meningioma. ${ }^{7}$ A large international study in 1178 glioma, meningioma and 2236 controls $330^{7}$ showed that there is an added risk for meningioma in men with a previous trauma and latency of 15-24 years, while the risk was very low or nonexistent for meningioma in women or gliomas in both genders. In our study, we could not find any correlation between trauma and a brain tumor, glioma or meningeoma.

Table I Incidence of primary brain tumors (per 100.000) secondary histology from 1993-2013

\begin{tabular}{llll}
\hline Year & Benign Tumor & Malign Tumor & Total Tumor \\
\hline 1993 & 3,4 & 4,8 & 8,2 \\
1994 & 3 & 4,6 & 7,6 \\
1995 & 3,1 & 4,7 & 7,8 \\
1996 & 4 & 6,1 & 10,1 \\
1997 & 3,1 & 4,4 & 7,5 \\
1998 & 3,3 & 4,5 & 7,8 \\
1999 & 3,6 & 4,8 & 8,4 \\
2000 & 3,8 & 5,2 & 9 \\
2001 & 4,2 & 6,1 & 10,3 \\
2002 & 4,5 & 6,6 & 11,1 \\
2003 & 4,8 & 6,8 & 11,6 \\
2004 & 5,1 & 7,1 & 12,2 \\
2005 & 5,2 & 7,2 & 12,4 \\
2006 & 5,1 & 8,1 & 13,2 \\
2007 & 5,5 & 8,4 & 13,9 \\
2008 & 5,2 & 8,5 & 13,7 \\
2009 & 5,8 & 8,8 & 14,6 \\
2010 & 5,6 & 9,2 & 14,8 \\
2011 & 5,8 & 9,4 & 15,2 \\
2012 & 5,7 & 9,2 & 14,9 \\
2013 & 6 & 9,4 & 15,4 \\
\hline
\end{tabular}

Table 2 Incidence of primary brain tumors secondary age group and histology from 1993-2013

\begin{tabular}{lllllllll}
\hline Age & GBM & Astro & Oligo & Meningeoma & Medulo/PNET & Ependimoma & Mix Glioma & Total \\
\hline $0-19$ & 0.4 & 0.8 & 0.2 & 0.01 & 0.7 & 0.4 & 0.06 & 5.1 \\
$20-34$ & 0.8 & 1.3 & 0.7 & 0.8 & 0.3 & 0.5 & 0.25 & 7.2 \\
$35-44$ & 1.8 & 2.1 & 0.9 & 2.5 & 0.15 & 0.6 & 0.2 & 9.3 \\
$45-54$ & 4.5 & 2.6 & 0.8 & 5.2 & 0.1 & 0.7 & 0.28 & 12.6 \\
$55-64$ & 8.1 & 3.8 & 0.7 & 7.1 & 0.05 & 0.55 & 0.3 & 14.1 \\
$65-74$ & 10.2 & 4.2 & 0.4 & 9.9 & 0.08 & 0.4 & 0.18 & 16.6 \\
$75-84$ & 9.8 & 3.9 & 0.2 & 11 & 0.04 & 0.3 & 0.1 & 17.5 \\
$85+$ & 7.7 & 2.4 & 0.06 & 10.9 & 0.03 & 0.2 & 0.07 & 16.2 \\
\hline
\end{tabular}

Table 3 Standart incidence (per x 100.000) secondary gender and histology from 1993-2013

\begin{tabular}{llll}
\hline Sex & Benign & Borderline & Malignant \\
\hline Males & 4 & 1.1 & 8.2 \\
Females & 7.2 & 1 & 6.4 \\
\hline
\end{tabular}

Table 4 Medical Risk Factors-exposure over 2 years

\begin{tabular}{ll}
\hline Infections & Conflicting results \\
\hline Alergy & low risk \\
Diabetes Mellitus & without effect \\
Cranial Trauma & poor effect \\
Hormonal Factors & menopauses increases the risk (glioma) \\
Skizofrenia, Depression, Meningitis & without effect \\
Epilepsy & Problematic connection \\
Cancer Family history & high risk \\
\hline
\end{tabular}


Low incidence of CNS tumors that makes even more difficult the resolution of the debate on the role of triggers brain tumors. In our study, a relatively small population and bias associated with having brain tumor may have altered the assessment of risk factors. Also, the group controls were not representative because they were mainly hospitalized in neurosurgery.

Low and high grade gliomas have different natural history reflected primarily through their age of presentation. This may involve different carcinogenic mechanisms probably influenced by the risk factors. We found in our study a correlation between head trauma and brain tumor (meningeoma) around 1\%, 9 male patients, not a correlation between glioma and traumatic brain injury (TBI) in male, and no correlation between meningeoma, glioma and TBI in female.

The role of epilepsy in development of brain tumors in our study is unclear, this for the stigma that exist in our country on epilepsy, only $0.87 \%$ ( 2 female, 6 male) of patients diagnosed with brain tumors referred to suffer from epilepsy from $10-15$ years. ${ }^{9-11}$

\section{Discussion}

From many times head trauma is suspected as medical risk factor for development of some kinds of brain tumors. Different studies have demonstrated the connection between head trauma and glioma, ${ }^{12}$ and head trauma and meningioma. International study with 1178 glioma and 330 meningeoma cases were individually or frequency matched to 2236 controls. ${ }^{7}$ Risk of having head injury was highest for meningioma in males, particularly when injury were sustained 15-24 years before diagnosis, ${ }^{7}$ while the risk for meningioma in females or gioma in both is lower or inexistent.

Epidemiological studies do not support a definitive connection between head injury and brain tumors and epilepsy and brain tumors. In our study, a relatively small population and bias associated with having brain tumor may have altered the assessment of risk factors. Also, the group controls were not representative because they were mainly hospitalized in neurosurgery. The retrospective nature of our study certainly increases the difficulty of evaluating risk factors, but although auto - reporting can identify the circumstances of exposure.

Despite this limitation in this study we found in our study a correlation between head trauma and brain tumor (meningeoma) around $1 \%$, not a correlation between glioma and traumatic brain injury (TBI) in male, and no correlation between meningeoma, glioma and TBI in female. The role of epilepsy in development of brain tumors in our study is unclear, this for the stigma that exist in our country on epilepsy, only $0.87 \%$ of patients diagnosed with brain tumors referred to suffer from epilepsy from 10-15 years.

\section{Acknowledgments}

None.

\section{Conflicts of interest}

None.

\section{References}

1. Arora RS, Alston RD, Eden TO, et al. Are reported increases of primary CNS tumors real? An analysis of longitudinal trends in England, $1979-$ 2003. Eur J Cancer. 2010;46(9):1607-1616

2. Porter KR, McCarthy BJ, Freels S, et al. Prevalence estimates for primary brain tumors in the United States by age, gender, behavior, and histology. Neuro Oncology. 2010;12(6):520-527

3. Wöhrer A, Waldhör T, Heinzl H, et al. The Austrian Brian Tumor Registry: a cooperative way to establish a population based brain tumor registry. J Neurooncology. 2009;95(3):401-411.

4. Bray F, Engholm G, Hakulinen T, et al. Trends in survival of patients diagnosed with cancers of the brain and nervous system, thyroid, eye, bone, and soft tissues in the Nordic countries 1964-2003 followed up until the end of 2006. Acta Oncologica. 2010;49(5):673-693.

5. Greig NH, Ries LG, Yancik R, et al. Increasing annual incidence of primary malignant brain tumors in the elderly. J Natl Cancer Inst. 1990;82(20):1621-1624.

6. Aldape K, Simmons ML, Davis RL, et al. Discrepancies in diagnoses of neuroepithelial neoplasms: The San Francisco Bay Area Adult Glioma Study. Cancer. 2000;88(10):2342-2349.

7. Preston-Martin S, Pogoda JM, Schlehofer B, et al. An international case-control study of adult glioma and meningioma: The role of head trauma. Int J Epidemiol. 1998;27(4):579-586.

8. Inskip PD, Linet MS, Heineman EF. Etiology of brain tumors in adults. Epidemiol Rev. 1995;17(1):382-414.

9. Schlehofer B, Blettner M, Preston-Martin S, et al. Role of medical history in brain tumour development: Results from the International Adult Brain Tumor Study. Int J Cancer. 1999;82(2):155-160.

10. Wiemels JL, Wiencke JK, Sison JD, et al. History of allergies among adults with glioma and controls. Int J Cancer. 2002;98(4):609-615.

11. Preston-Martin S. Epidemiology of primary CNS neoplasms. Neurol Clin. 1996;14(2):273-290.

12. Wrensch M, Miike R, Lee M, et al. Are prior head injuries or diagnostic $\mathrm{x}$-rays associated with glioma in adults? The effects of control selection bias. Neuroepidemiology. 2000;19(5):234-244. 